\title{
Neuroimmune Regulation of GABAergic Neurons Within the Ventral Tegmental Area During Withdrawal from Chronic Morphine
}

\begin{abstract}
Anna MW Taylor ${ }^{1,2}$, Annie Castonguay ${ }^{3,4}$, Atefeh Ghogha ${ }^{2}$, Pia Vayssiere ${ }^{2}$, Amynah AA Pradhan ${ }^{2,8}$, Lihua Xue ${ }^{5}$, Sadaf Mehrabani ${ }^{2}$, Juli Wu ${ }^{6}$, Pat Levitt ${ }^{6}$, Mary C Olmstead ${ }^{7}$, Yves De Koninck ${ }^{3,4}$, Christopher J Evans ${ }^{2}$ and Catherine M Cahill $*, 1,5$

'Department of Anesthesiology and Perioperative Care, University of California, Irvine, CA, USA; ${ }^{2}$ Hatos Center for Neuropharmacology, Semel Institute for Neuroscience and Human Behavior, University of California, Los Angeles, CA, USA; ' Institut Universitaire en Santé Mentale de Québec, Québec, QC, Canada; ${ }^{4}$ Department of Psychiatry and Neuroscience, Université Laval, Québec, QC, Canada; ${ }^{5}$ Department of Biomedical and Molecular Sciences, Queen's University, Kingston, ON, Canada; ${ }^{6}$ Children's Hospital Los Angeles and the Keck School of Medicine of the University of Southern California, Los Angeles, CA, USA; 'Department of Psychology, Queen's University, Kingston, ON, Canada
\end{abstract}

Opioid dependence is accompanied by neuroplastic changes in reward circuitry leading to a negative affective state contributing to addictive behaviors and risk of relapse. The current study presents a neuroimmune mechanism through which chronic opioids disrupt the ventral tegmental area (VTA) dopaminergic circuitry that contributes to impaired reward behavior. Opioid dependence was induced in rodents by treatment with escalating doses of morphine. Microglial activation was observed in the VTA following spontaneous withdrawal from chronic morphine treatment. Opioid-induced microglial activation resulted in an increase in brain-derived neurotrophic factor (BDNF) expression and a reduction in the expression and function of the $\mathrm{K}^{+} \mathrm{Cl}^{-}$co-transporter $\mathrm{KCC} 2$ within VTA GABAergic neurons. Inhibition of microglial activation or interfering with BDNF signaling prevented the loss of $\mathrm{Cl}^{-}$extrusion capacity and restored the rewarding effects of cocaine in opioid-dependent animals. Consistent with a microglial-derived BDNF-induced disruption of reward, intraVTA injection of BDNF or a KCC2 inhibitor resulted in a loss of cocaine-induced place preference in opioid-naive animals. The loss of the extracellular $\mathrm{Cl}^{-}$gradient undermines $\mathrm{GABA}_{\mathrm{A}}$-mediated inhibition, and represents a mechanism by which chronic opioid treatments can result in blunted reward circuitry. This study directly implicates microglial-derived BDNF as a negative regulator of reward in opioiddependent states, identifying new therapeutic targets for opiate addictive behaviors.

Neuropsychopharmacology (2016) 4I, 949-959; doi:I0.1038/npp.2015.221; published online I2 August 20I5

\section{INTRODUCTION}

Prescription, diversion, and illicit use of opioid therapeutics have emerged as a major problem in recent years (Compton and Volkow, 2006). A hallmark of opioid addiction is the high rate of relapse, which represents a significant clinical challenge. The propensity for dependent opioid abusers to relapse has been attributed to the dysphoria associated with drug abstinence that disrupts the ability to process rewards (Weiss and Koob, 2001; Goeldner et al, 2011).

Reduced activity within the mesocorticolimbic dopamine (DA) system is one element that contributes to a negative

\footnotetext{
*Correspondence: Dr CM Cahill, Department of Anesthesiology and Perioperative Care, University of California, 2117, Gillespie Neuroscience, 837 Health Science Road, Irvine, CA 92697, USA, Tel: + I 949824 5267, Fax: + I 949824 7066, E-mail: cmcahill@uci.edu

${ }^{8}$ Current address: Department of Psychiatry, University of Illinois, Chicago, I60I W Taylor Street, Chicago, IL 60612, USA.

Received 2 February 20 I5; revised I0 July 20 I5; accepted II July 20 I5; accepted article preview online 23 July 2015
}

affective state during withdrawal from drugs of abuse, such as opioids (Elman et al, 2013). This negative affect is proposed to contribute to pathological addictive behaviors (Koob and Le Moal, 2008). DA is the canonical neurotransmitter involved in motivated behavior and reward learning, and may contribute to the negative affective state during drug withdrawal. Striatal basal and evoked DA levels are significantly lower during withdrawal from chronic opioids (Acquas and Di Chiara, 1992; Zhang et al, 2009). Loss of striatal DA is linked to a deficit in spontaneous activity of DA neurons and failure of morphine to elicit the typical DA bursting activity following opioid withdrawal (Diana et al, 1995; Georges et al, 2006). Further evidence suggests that the loss of function of DA neurons in opioiddependent states has broader implications for general reward processing. For example, microdialysis studies found cocaine-induced DA release to be significantly attenuated following chronic opioid exposure (Leri et al, 2003). This loss of cocaine-stimulated extracellular DA levels is reflected in a reduction of spontaneous and drug-precipitated cocaine- 
seeking behavior (Stevenson et al, 2004; Leri et al, 2006). Brain-derived neurotrophic factor (BDNF) has been identified as being a critical modulator of VTA DA neurons in opioid-dependent animals (Koo et al, 2012; Vargas-Perez et al, 2014). Together, these studies indicate that the BDNFimpaired DA activity in opioid-dependent animals may undermine the ability of the VTA to elicit reward behaviors.

The current study identifies a novel neuroimmune mechanism by which opioid dependence interferes with mesolimbic DA function. We show that manipulation of BDNF or KCC2 can restore reward processing following chronic opioids, presenting a novel mechanism for therapeutic intervention in treating addictive behaviors mediated by dysphoric states.

\section{MATERIALS AND METHODS}

\section{Animals}

Male C57Bl/6 J and GAD65-GFP on C57Bl/6 background mice (The Jackson Laboratory, Bar Harbor, ME), 8-9-weeks old at the beginning of experimentation were used, unless a
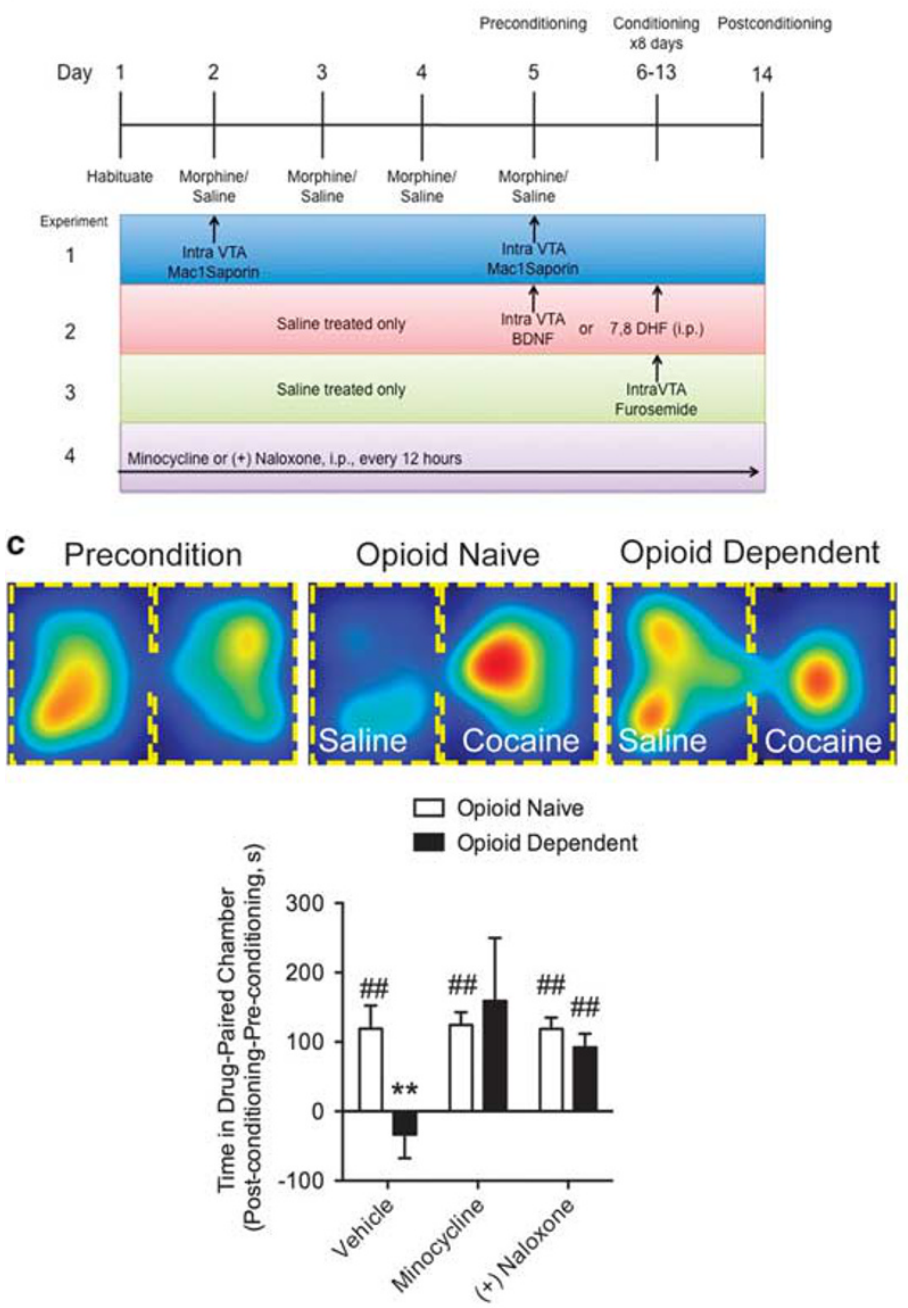

d

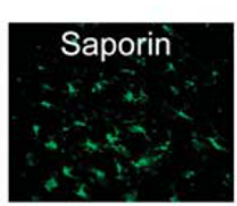

Mac1Sap

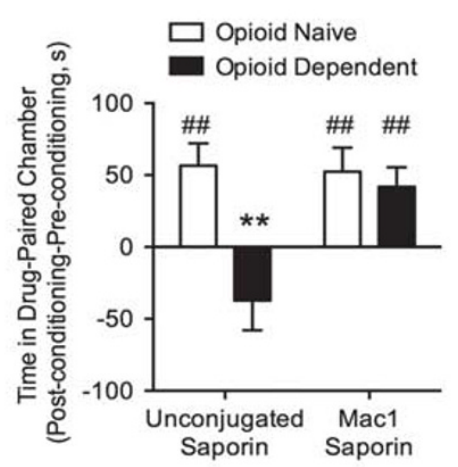

b
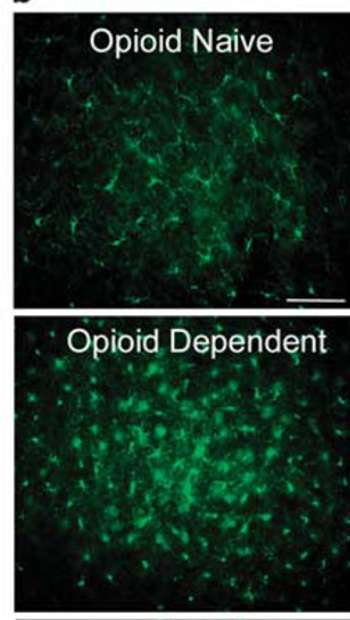

Dependent+MC
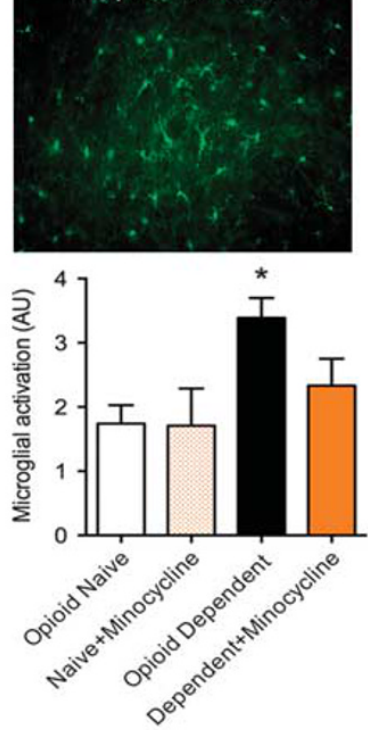
otherwise indicated. Male Sprague-Dawley rats (Charles River, QC) 8-9-weeks old were used in experiments with intra-cerebral drug administration, in order to assure precise administration of drug to the VTA. Animals were housed in groups of two to four until surgery, and kept on a 12-h light/ dark cycle with food and water available ad libitum. All behavioral tests were performed during the light phase. All procedures were conducted in accordance with the National Institutes of Health Guide for the Care of Use of Laboratory Animals and were approved by the UCLA Institutional Animal Care and Use Committee.

\section{Drug Treatment}

Mice were made opioid-dependent by twice-daily injections of escalating doses of morphine sulfate $(10,20,30,40 \mathrm{mg} / \mathrm{kg}$, intraperitoneal (i.p.); National Institutes on Drug Abuse (NIDA), Bethesda, MD) for 4 days. Control animals (opioidnaïve) received twice-daily vehicle (saline, $0.9 \% \mathrm{NaCl}$, i.p.) injections in lieu of the drug. To examine the impact of reducing the morphine-induced microglial response, a separate group of mice received twice-daily injections of minocycline hydrochloride $(30 \mathrm{mg} / \mathrm{kg}$, i.p.; Sigma, St Louis, $\mathrm{MO}$ ) or (+) naloxone (30 ng/kg, i.p.; NIDA) concomitant with morphine or saline beginning at the first morphine injection, until the end of the place preference protocol. Rats were made morphine-dependent by twice-daily injection of escalating doses of morphine sulfate $(5,8,10,10 \mathrm{mg} / \mathrm{kg}$, i.p.) for 4 days (Cahill et al, 2007). A control group received twice-daily injections of saline (as above) in lieu of the drug. For all molecular studies, animals were killed $12 \mathrm{~h}$ after last morphine or saline injection (Day 5) or immediately after the post-conditioning day (Day 14).

\section{Conditioned Place Preference (CPP)}

Systemic microglial inhibitors. The mouse CPP was conducted using an unbiased, counter balanced, twochamber apparatus (Supplementary Materials and Methods). Conditioning sessions consisted of mice receiving four trials of cocaine hydrochloride $(10 \mathrm{mg} / \mathrm{kg}$, i.p. NIDA) and four trials of saline, and were confined to the chambers for $15 \mathrm{~min}$. The CPP assay began $12 \mathrm{~h}$ after the last morphine injection, and hence, all conditioning sessions were completed when animals were in a state of spontaneous opioid withdrawal (Figure 1a). On the post-conditioning day, animals were allowed free access to both chambers in a drug-free state, and the time spent in the drug-paired chamber was measured over $15 \mathrm{~min}$. A preference score for each animal was calculated by taking the difference in time spent on the drug-paired chamber between the preconditioning and post-conditioning day.

Intra-VTA Mac-1-Saporin. The effect of specifically ablating microglia in the VTA on cocaine place preference in opioid-naive and -dependent animals was assessed. Rats were implanted with a bilateral cannula targeting the VTA (mm from bregma): AP -6.30, $\mathrm{ML}+/-0.8, \mathrm{DV}-8.0$. Immediately prior to chronic opioid or saline treatment, animals were injected with Mac-1-Saporin or unconjugated saporin (ATSBio; $0.25 \mathrm{ug} / 0.5 \mathrm{ul}$ per side). Animals were treated once again with Mac-1-Saporin or saporin immediately prior to the last injection of morphine. Twelve hours after the last opioid treatment, rats were conditioned to cocaine $(10 \mathrm{mg} / \mathrm{kg}$, i.p., as described above).

Intra-VTA furosemide. Rats were implanted with a bilateral intra-VTA cannula, as described above. Seven days after surgery, animals were treated with either furosemide (Sigma, $0.5 \mathrm{ul}$ of a $1 \mathrm{mM}$ in $0.5 \mathrm{ml} 0.1 \%$ DMSO in saline) or vehicle over $3 \mathrm{~min}$ and then immediately conditioned to cocaine or saline, as described above. Furosemide was injected prior to each conditioning session over 8 days. On the final day, animals were allowed free access to both conditioning chambers, and the time spent on the drug-paired side was assessed. Post hoc analysis of slices counterstained with cresyl violet was carried out to verify correct injection sites and to confirm that repeated injections did not lead to significant damage of the VTA.

Intra-VTA BDNF and systemic 7,8-dihydroxyflavone. Rats were implanted with a bilateral intra-VTA cannula, as described above. Seven days after surgery, the effect of intraVTA BDNF on cocaine CPP was assessed. In the BDNF group, BDNF (Sigma Aldrich; $25 \mathrm{ug}$ in $0.5 \mathrm{ul}$ saline) was injected into the VTA, over $3 \mathrm{~min}$. A separate vehicle group received saline injections of the same volume. One day after BDNF or saline injection, cocaine $(10 \mathrm{mg} / \mathrm{kg}$, i.p.) place preference was run, as described above.

Opioid-naïve rats received systemic 7,8-dihydroxyflavone injections (Tocris Bioscience, Bristol, UK, $10 \mathrm{mg} / \mathrm{kg}$, i.p.), a potent trkB agonist, $1 \mathrm{~h}$ prior to cocaine conditioning

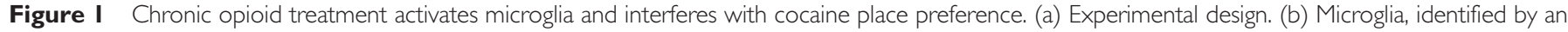

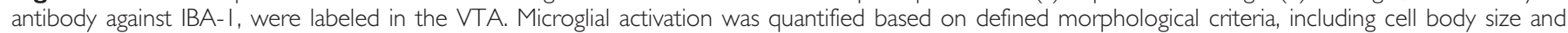

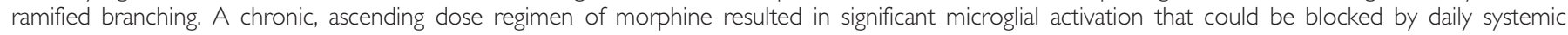

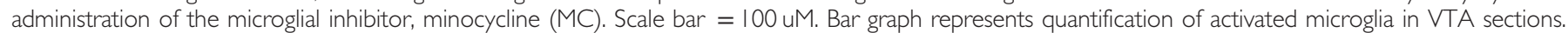

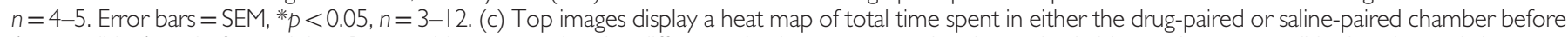

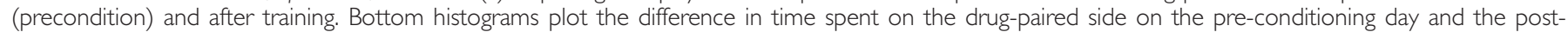

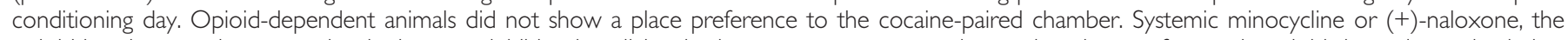

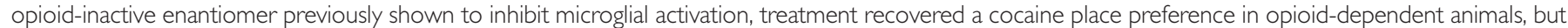

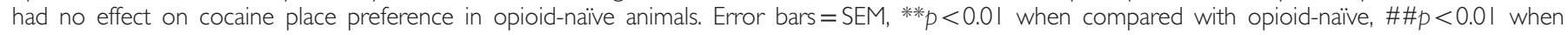

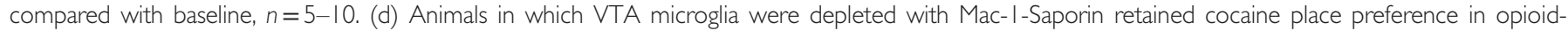

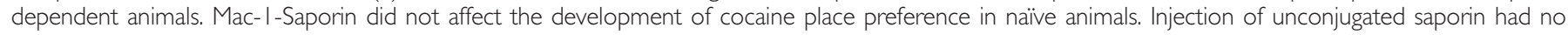

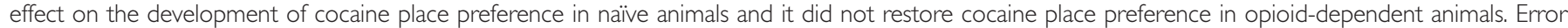

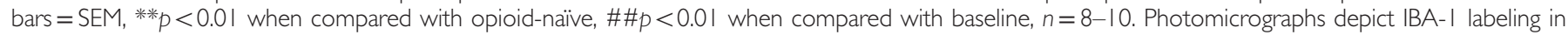
VTA sections from animals treated with unconjugated saporin or Mac-I-Saporin. Scale bar $=100$ uM.
} 
sessions. Control animals received vehicle (saline, i.p.) injections prior to cocaine conditioning sessions.

\section{Immunoblot Assay}

VTA brain punches from opioid-naïve and -dependent mice treated with or without minocycline were assayed for BDNF protein content using western blot (Supplementary Material and Methods). Equal amounts of protein ( $25 \mathrm{ug}$ ) were loaded onto pre-cast $10 \%$ polyacrylamide gels (Bio-Rad, Hercules, CA) and transferred to nitrocellulose membranes. Membranes were incubated with an antibody against BDNF (1:150; Millipore, Billerica, MA) and visualized with a goat anti-rabbit antibody conjugated to a horseradish peroxidase (HRP; Jackson Immunoresearch, West Grove, PA). Results were analyzed with a computer-assisted densitometry analysis (Image J Software, NIH).

\section{Immunocytochemistry}

Brain slices of the VTA were obtained from opioid-naïve and -dependent mice with or without minocycline treatment and prepared for immunocytochemical detection (Supplementary Material and Methods). For microglial staining, sections were incubated overnight with an antibody against IBA-1 (1:2000; Wako, Richmond, VA) at $4{ }^{\circ} \mathrm{C}$ followed by a goat anti-rabbit secondary antibody conjugated to Alexa Fluor 488 (1:1000, Millipore). For KCC2 staining, sections were incubated overnight with a rabbit antibody against KCC2 (1:500; Millipore) at $4{ }^{\circ} \mathrm{C}$ followed by a highly cross-adsorbed donkey anti-rabbit IgG conjugated to Alexa Fluor 594 (1:500; Invitrogen, Grand Island, NY). For KCC2 quantification, fluorescence intensity (total intensity per region of interest) was measured with Image J Software. For IBA1 quantification, the degree of microglial activation in the VTA was measured using a semi-quantitative method, based on defined morphological criteria, including cell body size, number of processes, and increasingly ramified morphology (Brettschneider et al, 2012). The level of microglial activation was scored on a linear scale (0-4) ranging from resting microglia (0) to highly activated (4).

\section{Fluorescent In situ Hybridization}

Opioid-naïve and -dependent mice were prepared for in situ labeling. Sections containing the VTA were labelled with triple fluorescent in situ hybridization using RNAscope (Advanced Cell Diagnostics, Hayward, CA, USA) following the manufacturer's protocol. Probes against BDNF, TH, $\mathrm{GAD}$, and integrin alpha $\mathrm{M}$ were used to label BDNF, DA neurons, GABAergic neurons, and microglia, respectively. Fluorescence signal intensity for each probe was quantified using ImageJ Software.

\section{Fluorescence Lifetime Imaging Microscopy}

Three-hundred-micrometer-thick coronal brain sections corresponding to the VTA of C57Bl/6 and GAD65-GFP knock-in mice were incubated in artificial cerebral spinal fluid (126 NaCl, $2.5 \mathrm{KCl}, 2 \mathrm{CaCl}_{2}, 2 \mathrm{MgCl}_{2}, 10$ glucose, 26 $\mathrm{NaHCO}_{3}, 1.25 \mathrm{NaH}_{2} \mathrm{PO}_{4}$ ) containing $5 \mathrm{mM}$ of the $\mathrm{Cl}^{-}$ indicator N-6-methoxyquinolinium acetoethylester (MQAE;
Invitrogen) for $30-40 \mathrm{~min}$ at $34^{\circ} \mathrm{C}$. MQAE fluorescence was excited using a Zeiss LSM510 laser-scanning microscope coupled to a femtosecond-pulsed Ti-Sapphire laser (Chameleon Ultra, Coherent, Santa Clara, CA) tuned at $750 \mathrm{~nm}$ (Gagnon et al, 2013). After a control period of $50 \mathrm{~s}$, the perfusion solution was switched to artificial cerebral spinal fluid containing $15 \mathrm{mM} \mathrm{KCl}$ (osmolarity adjusted using mannitol) to reverse $\mathrm{KCC} 2$-mediated $\mathrm{Cl}^{-}$transport to force $\mathrm{Cl}^{-}$accumulation inside the cell leading to a quenching of the measured lifetime (Chorin et al, 2011). Briefly, following the results of previous studies (Digman et al, 2008), we converted the photon timing histograms of each acquired lifetime image to phazer plots. To compare between groups, the average lifetime slope corresponding to the peak change in MQAE fluorescence was determined and compared. Means were calculated for each group ( $n=6-9$ animals) for analysis.

\section{Statistics}

For CPP, groups were compared with a Student's unpaired t-test or two-way ANOVA followed by post hoc analysis, where appropriate. A one-sample t-test was also run to determine whether the change in time spent in the drugpaired chamber was greater than baseline precondition times in the drug-assigned chamber (0). For the immunoblot assay, groups were compared with a Kruskal-Wallis test followed by a Dunn's post hoc analysis. For all other assays, groups were compared with a Student's unpaired t-test or one-way ANOVA followed by post hoc analysis, where appropriate. Results were considered statistically significant when $p<0.05$.

\section{RESULTS \\ Activated Microglia Mediate the Blunted Cocaine Reward in an Opioid-Dependent State}

We first identified cellular changes in the VTA following 4-day, twice-daily injection of escalating doses of morphine and spontaneous withdrawal. On the basis of IBA-1 immunostaining, we found microglia exhibited significant activation in the VTA, as measured by morphological analysis $\left(\mathrm{F}_{2,18}=5.49, p=0.01, n=3-12\right.$; Figure $\left.1 \mathrm{~b}\right)$. Microglia exhibited a stereotypical 'activated' phenotype, including an enlarged, compact cell body, thickening or hyperramification of cell processes, and greater number of immunolabeled cells compared with saline-treated animals. Opioid-induced microglial activation was attenuated by concomitant treatment with the microglial inhibitor minocycline. The morphology of microglia observed in animals treated with both chronic morphine and minocycline were indistinguishable from the ramified 'surveying' microglia observed in non-opioid-treated animals. Minocycline treatment in opioid-naïve animals did not produce any detectable changes in microglial morphology revealed by IBA-1 immunostaining.

To probe the function of the reward system following chronic morphine administration, we used cocaine reward, given the confounds of using an opioid to probe reward function in an opioid-receptor-desensitized state and that an 
opioid will alleviate withdrawal and could produce a negative reinforcement to drive reward-related behaviors. Blunted rewarding properties of cocaine have been observed previously following chronic morphine regimens (Stevenson et al, 2004; Leri et al, 2006), and we questioned whether microglia could play a role. In opioid-naïve animals, cocaine $(10 \mathrm{mg} / \mathrm{kg})$ induced a robust place preference, but, consistent with prior studies, failed to elicit a place preference in animals in an opioid-dependent state $\left(F_{\text {interaction }}(2,36)=3.41, p=0.04\right.$, $\mathrm{F}_{\text {microglial inhibitor }}(2,36)=3.67, \mathrm{p}=0.04, \mathrm{~F}_{\text {opioid treatment }}(1,36)=$ $2.38, p=0.13, n=5-10$; Figure 1c). We next tested the effect of inhibiting microglial activation on cocaine place preference in opioid-dependent mice. We used two different microglial inhibitors, minocycline and the inactive enantionmer opioid antagonist (+)-naloxone. (+)-Naloxone does not bind opioid receptors, but has been shown to specifically inhibit microglial activation (Iijima et al, 1978; Lewis et al, 2012; Wang et al, 2012; Mattioli et al, 2014). Remarkably, inhibiting microglia throughout the entire experimental period restored cocaine place preference in opioid-dependent animals (Figure 1c). In contrast, inhibition of microglial activation had no effect on cocaine preference in opioid-naïve animals.

We next examined whether microglia specifically within the VTA are required for the loss of cocaine place preference in opioid-dependent animals. Opioid-naïve and -dependent rats received microinjections of Mac-1-Saporin directly into the VTA to selectively destroy microglia within this region. Mac-1-Saporin microinjection restored cocaine place preference in opioid-dependent animals, and did not affect cocaine place preference in opioid-naïve animals $\left(\mathrm{F}_{\text {interaction }}(1,29)=5.92, \quad p=0.02, \mathrm{~F}_{\text {microglial inhibitor }}(1,29)=\right.$ 4.79, $p=0.04, \mathrm{~F}_{\text {opioid treatment }}(1,29)=9.29, p=0.005, \quad n=$ 8-10; Figure 1d). Control injection of unconjugated saporin did not affect cocaine place preference in either naïve or dependent animals (Figure 1d). Intra-VTA injection of Mac-1-Saporin or systemic treatment of opioids did not affect the amount of time spent in the neutral chamber on the post-conditioning day (average time in the neutral chamber was $261 \pm 29 \mathrm{~s}$ with no significant difference detected between groups).

Cocaine place preference is partially reliant on functional mu opioid receptors (Houdi et al, 1989; Kosten et al, 1991; Bilsky et al, 1992; Gerrits et al, 1995; Kuzmin et al, 1997). Because chronic morphine administration leads to the desensitization of $\mathrm{mu}$ opioid receptors (Williams et al, 2013), we tested whether microglial inhibition restored cocaine place preference via changes in mu opioid receptor function. Chronic opioid exposure led to significant reduction in $\mathrm{E}_{\max }$ of [D-Ala2, N-MePhe4, Gly-ol]-enkephalin (DAMGO)-stimulated $\left[{ }^{35} \mathrm{~S}\right] \mathrm{GTP} \gamma \mathrm{S}$ binding in the VTA, indicative of decreased functional $\mathrm{mu}$ opioid receptors within this region $(\mathrm{F}(2,12)=12.9, \quad p=0.001, n=4-6$; Supplementary Figure S1). Concomitant treatment of minocycline with escalating doses of morphine did not recover DAMGO-stimulated $\left[{ }^{35} \mathrm{~S}\right] \mathrm{GTP} \gamma \mathrm{S}$ binding, showing that the drug does not impact the general desensitization of mu opioid receptors following chronic morphine exposure.

\section{Activated Microglia in Opioid-Withdrawn Animals Release BDNF that Contributes to Blunted CPP}

We next performed a series of studies to elucidate the mechanism by which VTA-activated microglia contributes to blunted DA-mediated reward. We have previously reported that activated microglia release BDNF in the spinal cord after chronic morphine treatment (Ferrini et al, 2013). Together with the finding that BDNF serves as a negative modulator of opioid reward in the VTA (Koo et al, 2012), studies were performed to determine whether activated microglia-derived BDNF in the VTA mediates the blunted cocaine place preference in opioid-dependent animals.

Using fluorescent in situ hybridization analysis (RNAscope), BDNF mRNA levels were significantly elevated in the VTA of opioid-dependent animals $(\mathrm{t}(6)=7.69, p=0.03$, $n=4$; Figure 2a). Increased BDNF message was observed in microglia identified by co-expression of the integrin alpha $\mathrm{M}$ gene (itgam) (Figure $2 \mathrm{a}$ and $\mathrm{b}$ ). Smaller increases in BDNF were also observed in $\mathrm{TH}+$ cells, but not in GABAergic neurons (Supplementary Figure S2). BDNF protein was significantly elevated in the VTA of animals treated with chronic opioids (KW=14.92, $p=0.02, n=5$, Figure 2c). Pretreatment with minocycline blocked the rise in $\mathrm{BDNF}$ protein. These results suggest that although BDNF may be elevated in both neuronal and glial populations, inhibiting microglial activation in opioid-dependent animals is sufficient to reduce BDNF levels to resting levels.

We next examined whether BDNF was sufficient to block cocaine place preference in naïve animals. Opioid-naïve animals injected with BDNF directly into the VTA prior to place conditioning blocked the expression of cocaine place preference $(\mathrm{t}(8)=2.34, p=0.04, n=6-7$; Figure $2 \mathrm{~d})$. IntraVTA injection of BDNF did not affect the time spent in the neutral chamber on the post-conditioning day (Saline: $177.7 \pm 30.1 \mathrm{~s}$ vs BDNF: $218.7 \pm 26.1 \mathrm{~s})$. To demonstrate the VTA-specific effect of BDNF-induced blunting of place preference, naïve animals were treated systemically with the TrkB agonist, 7,8-dihydroxyflavone. Treatment with a systemic TrkB agonist produced an increase in the magnitude of cocaine place preference $(\mathrm{t}(13)=2.51$, $p=0.03, n=7-8$; Figure 2d), an opposite effect to that of local VTA administration of BDNF. These results support the finding that $\mathrm{BDNF}$ arising via microglial activation in the VTA of opioid-dependent animals is sufficient to block cocaine place preference.

\section{Withdrawal from Chronic Opioids Leads to Impaired $\mathrm{Cl}^{-}$ Transport Associated with a Loss of KCC2 Expression in VTA GABAergic Neurons}

We next explored the mechanism by which BDNF may modulate VTA neuronal activity in opioid-dependent states. In the adult hippocampus and spinal cord, BDNF causes a downregulation of the $\mathrm{K}^{+} / \mathrm{Cl}^{-}$co-transporter, $\mathrm{KCC} 2$ (Rivera et al, 2002; Coull et al, 2005; Ferrini et al, 2013). KCC2 is pivotal in maintaining the $\mathrm{Cl}^{-}$gradient to enable $\mathrm{GABA}_{\mathrm{A}}-$ mediated hyperpolarization (Fiumelli et al, 2005). A previous study found that an acute opioid challenge in opioiddependent animals potentiated, rather than inhibited, GABAergic currents in VTA GABAergic neurons, suggesting $B D N F$ may have a specific effect on $G_{A B A}$ function 
a
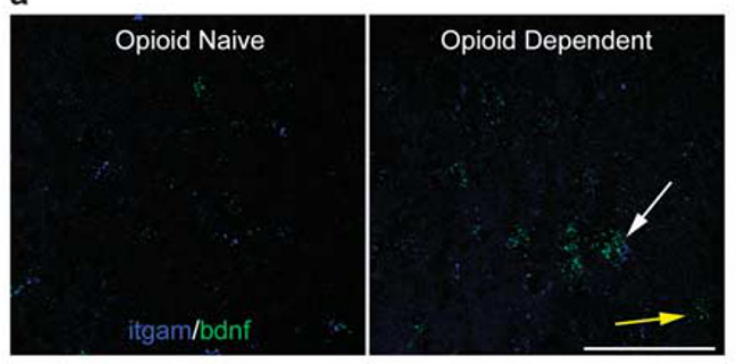

b

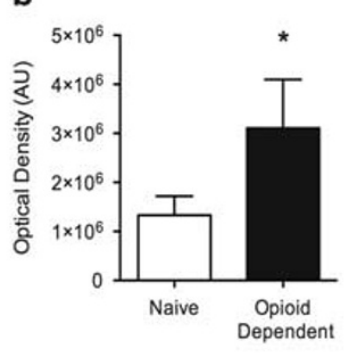

C

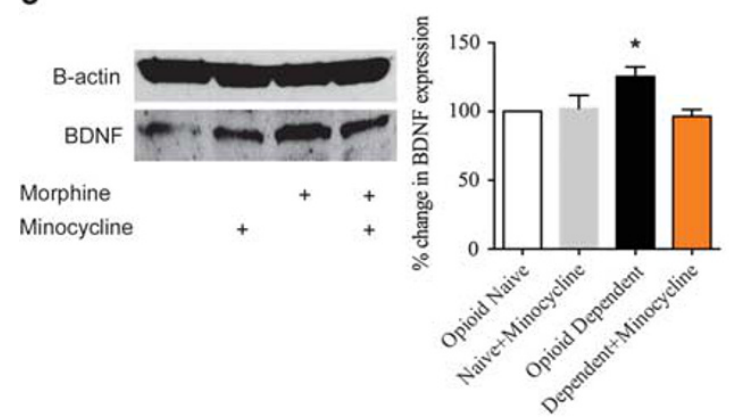

d

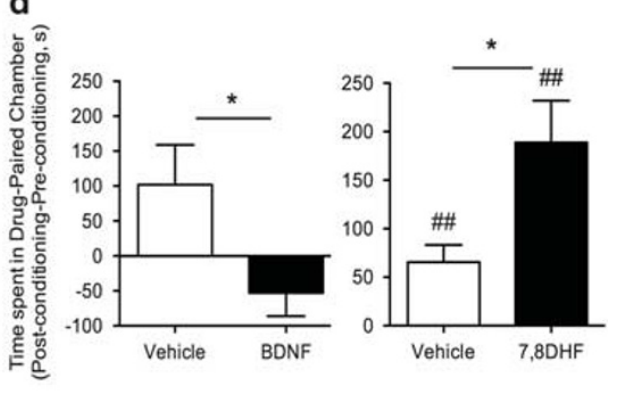

Figure 2 Chronic opioid treatment stimulates BDNF expression in the VTA that contributes to a blunted cocaine place preference. (a) BDNF mRNA (green) was detected with fluorescent in situ hybridization in both microglial (blue, identified with itgam promoter; white arrow) and non-microglial cells (yellow arrow). Chronic morphine caused an increase in BDNF transcripts in the VTA sections from opioid-dependent animals. (b) Total BDNF mRNA in VTA sections was semi-quantified using image analysis (see methods), and BDNF levels were significantly elevated in the VTA of opioid-dependent animals. Error bars $=$ SEM, $* p<0.05, n=4$. (c) Tissue punches from the VTA were analyzed for BDNF protein expression using western blot analysis. Opioiddependent animals showed an increase in BDNF protein (normalized to beta-actin) that was significantly greater than opioid-naïve brains. Minocycline reduced BDNF expression in the VTA of opioid-dependent animals. Error bars $=S E M, * p<0.05, n=5$. (d) Bilateral infusion of BDNF into the VTA of opioid-naïve animals prior to cocaine conditioning blocked the expression of cocaine place preference. Systemic injection with a potent trkB agonist, 7,8-dihydroxyflavone, enhanced cocaine CPP in opioid-naïve animals, compared with animals treated with vehicle. Error bars $=$ SEM, *p $<0.05$ when compared with vehicle, $\# \# p<0.01$, when compared with baseline, $n=6-8$.

(Madhavan et al, 2010). We first examined changes in KCC2 expression in the VTA of opioid-dependent animals. Quantitative analysis of KCC2 immunolabeling in the VTA of opioid-naïve and -dependent tissue confirmed KCC2 downregulation in opioid-dependent states when measured $12 \mathrm{~h}$ after final morphine injection (day 5) $(\mathrm{t}(22)=2.87$, $p=0.009, n=9-12$; Figure $3 \mathrm{~b})$. KCC2 expression remained low throughout the cocaine CPP in opioid-dependent animals (Day 14). Further, KCC2 expression was found exclusively on non-TH positive neurons (Figure $3 \mathrm{a}$ ). We next investigated changes in $\mathrm{Cl}^{-}$transport rate in VTA GABAergic neurons opioid-dependent states. The rate of $\mathrm{Cl}^{-}$ transport was measured using a fluorescent $\mathrm{Cl}^{-}$indicator, MQAE, and a reverse transport strategy using abrupt elevation of extracellular $\mathrm{K}^{+}$. We have used this method previously to measure $\mathrm{Cl}^{-}$transport rate within spinal cord neurons (Doyon et al, 2011; Ferrini et al, 2013). The MQAE dye loaded exclusively in GABAergic neurons, identified by GAD co-labeling in sections taken from transgenic GAD65GFP mice (Figure 3c). We found that opioid-dependent animals in a state of withdrawal displayed a significantly slower rate of $\mathrm{Cl}^{-}$transport $(\mathrm{F}(3,24)=0.25, p=0.02, n=7-9$; Figure $3 e$ and $\mathrm{f}$ ). Application of a $\operatorname{TrkB}$ function-blocking antibody directly onto the VTA slices harvested from morphine-dependent animals restored normal $\mathrm{Cl}^{-}$transport rate. These data show that BDNF has a critical role in mediating the chronic opioid-induced dysregulation of $\mathrm{Cl}^{-}$ homeostasis in VTA GABAergic neurons. Application of a KCC2 inhibitor, furosemide, to naïve slices inhibited $\mathrm{Cl}^{-}$ transport, reminiscent of the opioid-dependent state. Importantly, intra-VTA injection of furosemide blocked cocaine CPP in opioid-naïve animals $(\mathrm{t}(12)=2.41, p=0.03$, $n=6-8$, Figure $3 g$ ). Furosemide treatment did not affect the time spent in the neutral compartment on the postconditioning day (Saline: $352.5 \pm 39.1 \mathrm{~s}$ vs Furosemide: $391.4 \pm 43.4 \mathrm{~s}$ ). This directly implicates the loss of KCC2 function in the VTA in driving blunted cocaine reward (Figure 4).

\section{DISCUSSION}

\section{Increased VTA GABAergic Activity in Opioid-Dependent States}

The present study provides the first evidence that microgliaderived BDNF can mediate the blunted DA activity in opioid-dependent and withdrawn states. Here, we show chronic opioid treatment causes dysregulation in transmembrane $\mathrm{Cl}^{-}$homeostasis in GABAergic neurons of the VTA driven by BDNF and activated microglia.

DA neuronal activity is tonically inhibited by GABAergic VTA interneurons and extrinsic GABAergic projections. Activation of $\mathrm{GABA}_{\mathrm{A}}$ receptors hyperpolarizes these inhibitory neurons and stimulates DA release (disinhibition). 
a
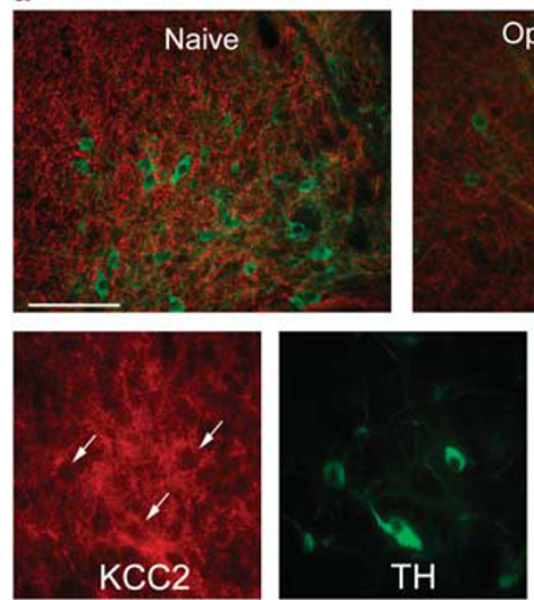

C
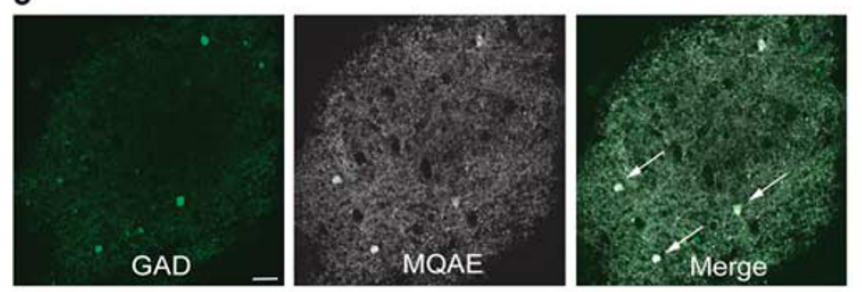

e

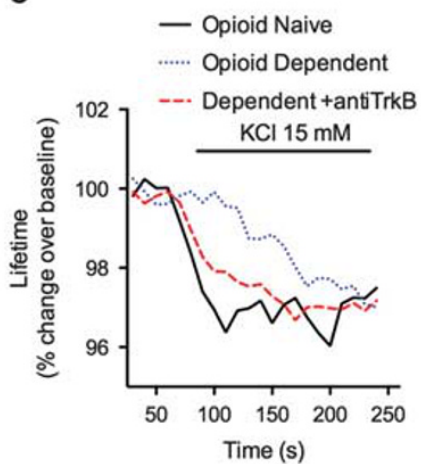

f

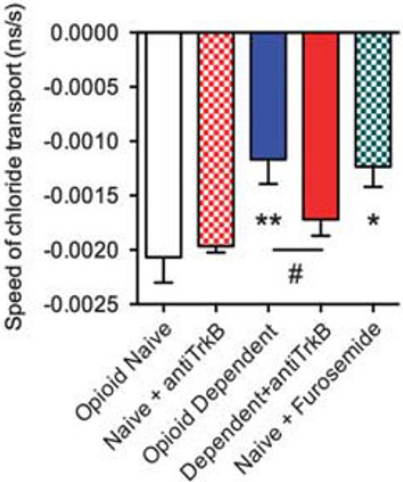

b

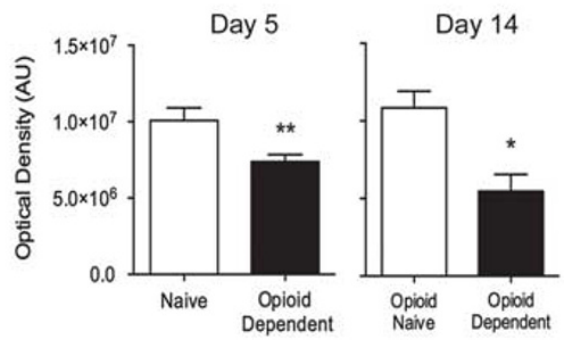

d

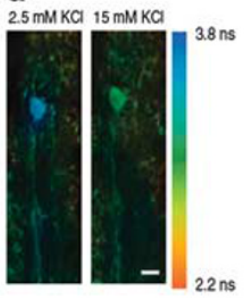

g

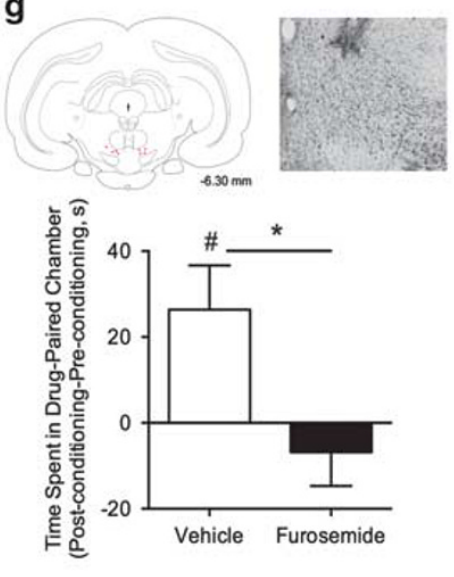

Figure 3 Decreased $\mathrm{Cl}^{-}$transport in GABAergic VTA neurons related to a loss KCC2 expression. (a) Top images show KCC2 expression (red) surrounding tyrosine hydroxylase (TH)-positive neurons (green) in the VTA is significantly decreased after chronic morphine exposure (opioid-dependent). Images were taken at $\times 20$ magnification. Scale $=75 \mu \mathrm{m}$. Bottom images show KCC2 expression mainly found in non-TH+ neurons. White arrowhead indicates region of $\mathrm{TH}+$ neuron with low $\mathrm{KCC} 2$ expression. Images were taken at $\times 40$ magnification. Scale $=50 \mu \mathrm{m}$. (b) Histogram depicts quantification of KCC2 labeling in the VTA of opioid-naïve and -dependent animals either $12 \mathrm{~h}$ after final morphine injection (Day 5) or after cocaine conditioned place preference (CPP) (Day 14). Opioid-dependent animals showed a significant reduction in KCC2 expression in the VTA. Error bars $=$ SEM, * $p<0.05$, *** $p<0.01, n=9-12$. (c) VTA slices from control animal showing MQAE (white) loaded exclusively in GAD+ neurons (green). White arrowheads indicate $\mathrm{GAD}+$ neurons loaded with MQAE. Scale $=20 \mu \mathrm{m}$. (d) Pseudocolor images showing lifetime maps from control VTA slices in the presence of 2.5 or $15 \mathrm{mM}$ $\mathrm{KCl}^{-}$. (e) Representative MQAE lifetime plots. VTA slices from opioid-dependent animals showed slower rate of $\mathrm{Cl}^{-}$transport into the cell, as measured by time to quench fluorescence of the $\mathrm{Cl}^{-}$indicator, MQAE after an abrupt chance in extracellular $\mathrm{K}+$ to reverse $\mathrm{Cl}^{-}$transport. Acute treatment with the TrkB antagonist restored $\mathrm{Cl}^{-}$transport. (f) Average slope at the 50-s time point for all groups. Movement of $\mathrm{Cl}^{-}$into the cell was confirmed to be through $\mathrm{KCC} 2$ by application of a KCC2 antagonist, furosemide, which attenuated the change in MQAE fluorescence lifetime. $n=7-9$, error bars $=$ SEM * $p<0.05$, *** $p<0.0$, compared with opioid-naïve group and \#p<0.05, compared with opioid-dependent group, $n=7-9$. (g) The KCC2 inhibitor, furosemide, injected directly into the VTA prior to cocaine conditioning, blocked cocaine CPP in opioid-naive animals. Error bars $=\mathrm{SEM}, * p<0.05$ compared with vehicle, \#p $<0.05$ when compared with baseline, $n=6-8$. Left inset indicates injection sites $(6.30 \mathrm{~mm}$ posterior to bregma) and right inset is a representative image of an injection site counterstained with cresyl violet ( $\times 5$ magnification). 


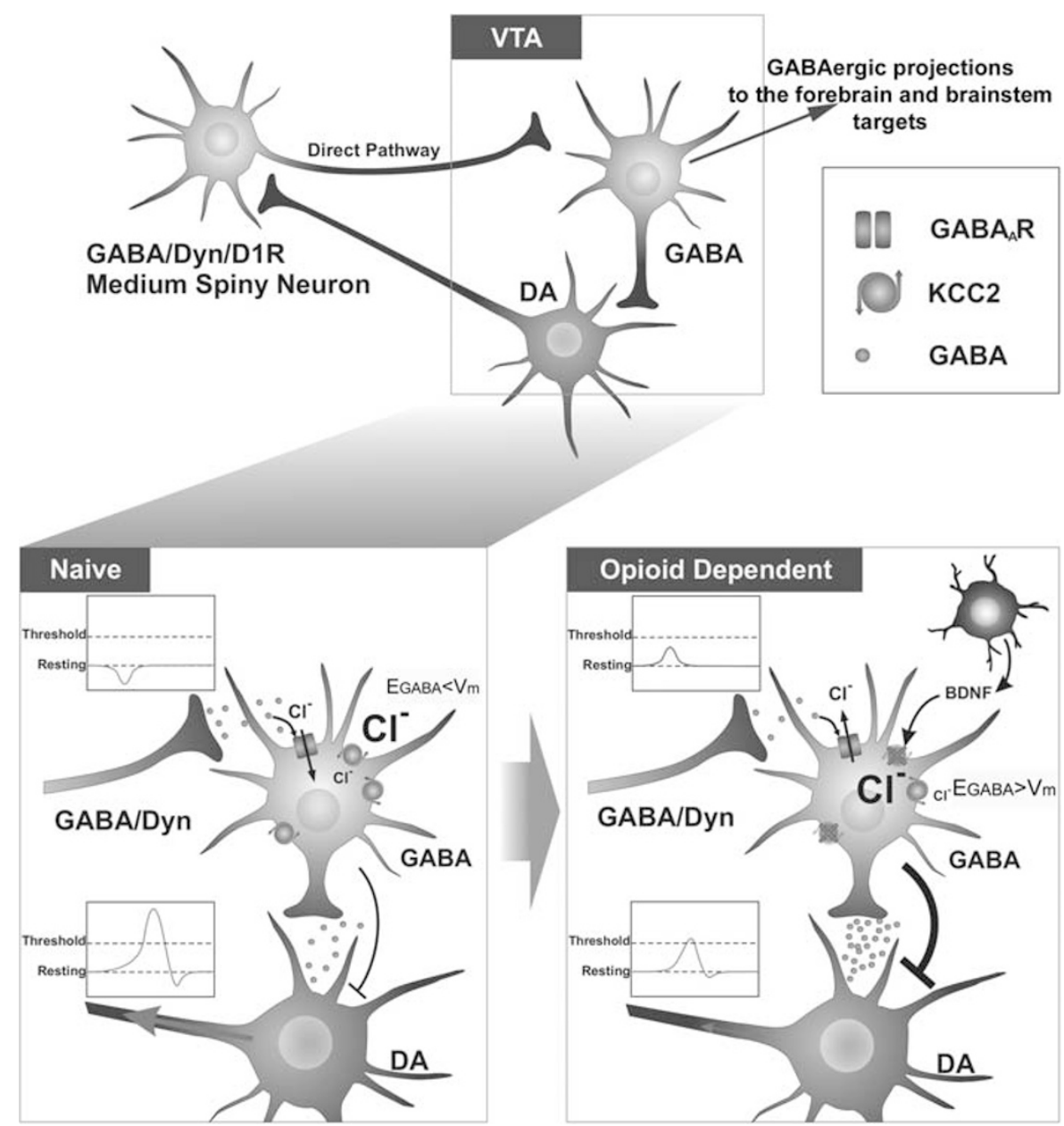

Figure 4 Normally, cocaine-stimulated DA in NAc activates reciprocal GABAergic medium spiny neurons (MSN) that project onto GABAergic VTA neurons. While GABAergic VTA neurons project to a range of forebrain and brainstem targets, cocaine mediated inhibition of GABAergic interneurons causes a net increase in VTA DA activity and further stimulates the release of DA in the NAc. In opioid-dependent states, KCC2 expression in GABAergic neurons of the VTA is mediated by BDNF. Decreased KCC2 expression leads to a dysregulation of the $\mathrm{Cl}^{-}$gradient, compromising the inhibitory potential of these neurons. Release of GABA from the MSN projection neurons is less inhibitory on these neurons, leading to an increased excitability of GABAergic VTA neurons, and increased inhibition of DA neurons. Cocaine is no longer able to stimulate DA release in the NAc, and CPP is significantly impaired.

KCC2 normally transports $\mathrm{Cl}^{-}$out of GABAergic neurons to maintain a low intracellular concentration and is critical for maintaining the inhibitory potential of $\mathrm{GABA}_{\mathrm{A}}$ receptors (Rivera et al, 2002; Viitanen et al, 2010). While both DA and GABAergic neurons of the VTA express $\mathrm{GABA}_{\mathrm{A}}$ receptors (Churchill et al, 1992; Kalivas, 1993), DA cell bodies do not express immunoreactive or functional KCC2, and appear to use alternative $\mathrm{Cl}^{-}$transporters, such as $\mathrm{Na}$-dependent anion exchanger, to maintain the $\mathrm{Cl}^{-}$gradient (Gulacsi et al, 2003). Therefore, downregulation of KCC2 in the VTA will disrupt $\mathrm{GABA}_{\mathrm{A}}$ signaling in GABAergic rather than DA neurons. In the present study, GABAergic neurons of opioiddependent animals exhibit a compromised $\mathrm{Cl}^{-}$transport activity paralleled by a loss of KCC2 expression. Further, our data demonstrate that directly inhibiting KCC2 transport in naïve animals mimics the opioid-dependent state by blocking VTA $\mathrm{Cl}^{-}$transport and cocaine CPP. Loss of KCC2 function leads to an accumulation of intracellular $\mathrm{Cl}^{-}$within the cell and a reduction in $\mathrm{Cl}^{-}$influx upon $\mathrm{GABA}_{\mathrm{A}}$ receptor activation, resulting in a reduction in GABA-mediated hyperpolarization (Rivera et al, 1999; Hubner et al, 2001;
Coull et al, 2003; Laviolette and van der Kooy, 2004; Taylor et al, 2015). These data support previous studies demonstrating that KCC2 inhibition compromises the inhibitory effect of a $\mathrm{GABA}_{\mathrm{A}}$ agonist in the VTA (Ting et al, 2013). Our model predicts that decreased $\mathrm{GABA}_{\mathrm{A}}$-mediated inhibition onto GABAergic neurons would increase inhibitory tone onto VTA DA neurons in opioid-dependent states. Decreased DA neuronal activity in opioid-dependent states is a robust finding in multiple studies using electrophysiological, neurochemical, and behavioral approaches (Leri et al, 2003, 2006; Stevenson et al, 2004; Georges et al, 2006). A shift in $\mathrm{GABA}_{\mathrm{A}}$-mediated inhibition on GABAergic neurons is also supported by the observation that intra-VTA DAMGO potentiated, rather than inhibited, GABAergic currents on DA neurons in opioid-dependent animals (Madhavan et al, 2010). Further, the amplitude and frequency of spontaneous miniature $\mathrm{GABA}_{\mathrm{A}}$ inhibitory postsynaptic potentials recorded from DA cells were greater in opioid-dependent animals in acute withdrawal, suggestive of increased GABA release onto DA neurons in opioid-dependent animals (Bonci and Williams, 1997). 
In addition to the GABAergic interneurons, VTA GABAergic neurons project to a wide range of targets outside the midbrain, including the prefrontal cortex, hypothalamus, and habenula (Carr and Sesack, 2000; Taylor et al, 2014). Our current study cannot differentiate between these GABAergic populations, and it remains to be seen whether other behavioral phenotypes driven by these GABAergic projection neurons are also disrupted in opioid-dependent states.

\section{Changes in VTA GABAergic Activity is Mediated by BDNF and Activated Microglia}

Activated microglia are known to modify opioid function. Chronic opioids activate glia in the spinal cord and blocking microglial activation prolongs the effectiveness of morphine analgesia and prevents the development of opioid-induced hyperalgesia (Raghavendra et al, 2004; Cui et al, 2006; Hutchinson et al, 2009; Ferrini et al, 2013). Activated microglia release BDNF, which acts as a modulator of neuronal activity. Release of BDNF from activated microglia in the spinal cord also results in a loss of GABAergic inhibition relating to a dysregulation of the transmembrane $\mathrm{Cl}^{-}$gradient (Ferrini et al, 2013). There is also evidence that activated microglia contribute to supraspinal opioid modulation. Chronic opioid exposure results in activated microglia in supraspinal regions, such as the nucleus accumbens and VTA (Hutchinson et al, 2009). Blocking microglial activation suppresses morphine-induced respiratory depression and some measures of morphine-induced reward (Hutchinson et al, 2008). The current study directly implicates activated microglia in the VTA driving aberrant cocaine reward behavior in opioid-dependent states by interfering with GABAergic inhibition via the BDNF-KCC2 pathway. We have also shown that inhibition of microglial activation, either systemically or directly within the VTA, is sufficient to restore cocaine reward behavior in opioid-dependent animals (Figure 1).

VTA neurons express BDNF and TrkB receptors (Gall et al, 1992; Seroogy et al, 1994), and several studies suggest BDNF acts as a critical modulator of DA circuitry within the VTA. For example, injecting BDNF into the VTA decreases morphine place preference and blocks morphine-stimulated burst firing of DA neurons (Koo et al, 2012). In the same study, localized knockdown of BDNF in the VTA enhances the acute rewarding effect of morphine. Evidence that BDNF mediates the impaired DA signaling in opioid dependence includes injection of BDNF into the VTA, which mimicked an opioid-dependent reward-like state (Vargas-Perez et al, 2009). In our current study, we have shown that chronic morphine leads to increased total VTA BDNF protein levels as well as increased BDNF mRNA levels in VTA microglia. Selective injection of BDNF into the VTA blocked cocaine reward in opioid-dependent animals. These results support and build upon previously published work that suggests VTA $\mathrm{BDNF}$ acts as a negative modulator of drug reward (Koo et al, 2012). However, prior studies have shown hippocampal BDNF to facilitate learning and memory in spatial tasks, such as the CPP (Leal et al, 2014). In addition, direct injection of $\mathrm{BDNF}$ into the NAc has been shown to potentiate some elements of cocaine reward (Horger et al, 1999; Guillin et al, 2001; Graham et al, 2007). Indeed, we found that systemic injection of the BDNF agonist, 7,8-dihydroxyflavone, in naïve animals facilitated cocaine place preference (Figure 2d). This suggests that the enhanced learning effect of BDNF mediated through the hippocampus and other regions is the predominant effect. However, the present study as well as previous studies (Vargas-Perez et al, 2009; Koo et al, 2012) have shown BDNF injected directly into the VTA impairs mesolimbic DA activity and reward learning, suggesting BDNF has a varied and circuit-specific effect. How, or if, the role of BDNF in reward learning changes throughout the progression of other drug-dependent states remains to be explored, but some studies suggest the role of VTA BDNF may be altered in cocaine dependence ( $\mathrm{Lu}$ et al, 2004).

We have identified a novel adaptive mechanism following chronic opioid exposure that involves microglial-BDNFTrkB-KCC2 signaling augmenting the inhibitory GABAergic tone within the VTA. This cascade of events leads to an overall increased inhibition on VTA DA output neurons. Restoration of the $\mathrm{Cl}^{-}$gradient in GABAergic neurons, either through targeting KCC2 activity or through prevention of microglial activation, may be useful new strategies for preventing and perhaps recovering from some of the reward-related behavioral deficits associated with chronic opioid use.

\section{FUNDING AND DISCLOSURE}

We would like to acknowledge the NIDA Drug Supply Program for providing the (+) naloxone used in this study. Financial support was provided by the Shirley and Stefan Hatos Foundation (AMWT, AG; CJE is the holder of the Stefan and Shirley endowed chair), and Canadian Institutes of Health Research grant MOP 12942 (YDK) and MOP 123298 (CMC, MCO). The authors declare no conflict of interest.

\section{ACKNOWLEDGMENTS}

The authors would like to thank the California Nanosystems Institute Advanced Light Microscopy/Spectroscopy core at UCLA for technical assistance. We would like to thank Sarah Pickens and Edmund Ong for technical assistance and for constructive comments on earlier versions of this manuscript. We would also like to thank Churmy Yong Fan for her artistic design.

\section{REFERENCES}

Acquas E, Di Chiara G (1992). Depression of mesolimbic dopamine transmission and sensitization to morphine during opiate abstinence. J Neurochem 58: 1620-1625.

Bilsky EJ, Montegut MJ, Delong CL, Reid LD (1992). Opioidergic modulation of cocaine conditioned place preferences. Life Sci 50: Pl85-P190.

Bonci A, Williams JT (1997). Increased probability of GABA release during withdrawal from morphine. J Neurosci 17: 796-803.

Brettschneider J, Toledo JB, Van Deerlin VM, Elman L, McCluskey L, Lee VM et al (2012). Microglial activation correlates with disease progression and upper motor neuron clinical symptoms in amyotrophic lateral sclerosis. PloS ONE 7: e39216. 
Cahill CM, Holdridge SV, Morinville A (2007). Trafficking of deltaopioid receptors and other G-protein-coupled receptors: implications for pain and analgesia. Trends Pharmacol Sci 28: 23-31.

Carr DB, Sesack SR (2000). GABA-containing neurons in the rat ventral tegmental area project to the prefrontal cortex. Synapse 38: $114-123$.

Chorin E, Vinograd O, Fleidervish I, Gilad D, Herrmann S, Sekler I et al (2011). Upregulation of KCC2 activity by zinc-mediated neurotransmission via the mZnR/GPR39 receptor. J Neurosci 31: 12916-12926.

Churchill L, Dilts RP, Kalivas PW (1992). Autoradiographic localization of gamma-aminobutyric acid A receptors within the ventral tegmental area. Neurochem Res 17: 101-106.

Compton WM, Volkow ND (2006). Major increases in opioid analgesic abuse in the United States: concerns and strategies. Drug Alcohol Depend 81: 103-107.

Coull JA, Beggs S, Boudreau D, Boivin D, Tsuda M, Inoue K et al (2005). BDNF from microglia causes the shift in neuronal anion gradient underlying neuropathic pain. Nature 438: 1017-1021.

Coull JA, Boudreau D, Bachand K, Prescott SA, Nault F, Sik A et al (2003). Trans-synaptic shift in anion gradient in spinal lamina I neurons as a mechanism of neuropathic pain. Nature 424: 938-942.

Cui Y, Chen Y, Zhi JL, Guo RX, Feng JQ, Chen PX (2006). Activation of p38 mitogen-activated protein kinase in spinal microglia mediates morphine antinociceptive tolerance. Brain Res 1069: 235-243.

Diana M, Pistis M, Muntoni A, Gessa G (1995). Profound decrease of mesolimbic dopaminergic neuronal activity in morphine withdrawn rats. J Pharmacol Exp Ther 272: 781-785.

Digman MA, Dalal R, Horwitz AF, Gratton E (2008). Mapping the number of molecules and brightness in the laser scanning microscope. Biophys J 94: 2320-2332.

Doyon N, Prescott SA, Castonguay A, Godin AG, Kroger H, De Koninck Y (2011). Efficacy of synaptic inhibition depends on multiple, dynamically interacting mechanisms implicated in chloride homeostasis. PLoS Comput Biol 7: e1002149.

Elman I, Borsook D, Volkow ND (2013). Pain and suicidality: insights from reward and addiction neuroscience. Prog Neurobiol 109: 1-27.

Ferrini F, Trang T, Mattioli TA, Laffray S, Del'Guidice T, Lorenzo LE et al (2013). Morphine hyperalgesia gated through microglia-mediated disruption of neuronal $\mathrm{Cl}(-)$ homeostasis. Nat Neurosci 16: 183-192.

Fiumelli H, Cancedda L, Poo MM (2005). Modulation of GABAergic transmission by activity via postsynaptic Ca2+dependent regulation of KCC2 function. Neuron 48: 773-786.

Gagnon M, Bergeron MJ, Lavertu G, Castonguay A, Tripathy S, Bonin RP et al (2013). Chloride extrusion enhancers as novel therapeutics for neurological diseases. Nat Med 19: 1524-1528.

Gall CM, Gold SJ, Isackson PJ, Seroogy KB (1992). Brain-derived neurotrophic factor and neurotrophin-3 mRNAs are expressed in ventral midbrain regions containing dopaminergic neurons. $\mathrm{Mol}$ Cell Neurosci 3: 56-63.

Georges F, Le Moine C, Aston-Jones G (2006). No effect of morphine on ventral tegmental dopamine neurons during withdrawal. J Neurosci 26: 5720-5726.

Gerrits MA, Patkina N, Zvartau EE, van Ree JM (1995). Opioid blockade attenuates acquisition and expression of cocaineinduced place preference conditioning in rats. Psychopharmacology 119: 92-98.

Goeldner C, Lutz PE, Darcq E, Halter T, Clesse D, Ouagazzal AM et al (2011). Impaired emotional-like behavior and serotonergic function during protracted abstinence from chronic morphine. Biol Psychiatry 69: 236-244.

Graham DL, Edwards S, Bachtell RK, DiLeone RJ, Rios M, Self DW (2007). Dynamic BDNF activity in nucleus accumbens with cocaine use increases self-administration and relapse. Nat Neurosci 10: 1029-1037.

Guillin O, Diaz J, Carroll P, Griffon N, Schwartz JC, Sokoloff P (2001). BDNF controls dopamine D3 receptor expression and triggers behavioural sensitization. Nature 411: 86-89.

Gulacsi A, Lee CR, Sik A, Viitanen T, Kaila K, Tepper JM et al (2003). Cell type-specific differences in chloride-regulatory mechanisms and $\mathrm{GABA}(\mathrm{A})$ receptor-mediated inhibition in rat substantia nigra. J Neurosci 23: 8237-8246.

Horger BA, Iyasere CA, Berhow MT, Messer CJ, Nestler EJ, Taylor JR (1999). Enhancement of locomotor activity and conditioned reward to cocaine by brain-derived neurotrophic factor. J Neurosci 19: 4110-4122.

Houdi AA, Bardo MT, Van Loon GR (1989). Opioid mediation of cocaine-induced hyperactivity and reinforcement. Brain Res 497 : 195-198.

Hubner CA, Stein V, Hermans-Borgmeyer I, Meyer T, Ballanyi K, Jentsch TJ (2001). Disruption of KCC2 reveals an essential role of $\mathrm{K}-\mathrm{Cl}$ cotransport already in early synaptic inhibition. Neuron $\mathbf{3 0}$ : $515-524$.

Hutchinson MR, Lewis SS, Coats BD, Skyba DA, Crysdale NY, Berkelhammer DL et al (2009). Reduction of opioid withdrawal and potentiation of acute opioid analgesia by systemic AV411 (ibudilast). Brain Behav Immun 23: 240-250.

Hutchinson MR, Northcutt AL, Chao LW, Kearney JJ, Zhang Y, Berkelhammer DL et al (2008). Minocycline suppresses morphine-induced respiratory depression, suppresses morphineinduced reward, and enhances systemic morphine-induced analgesia. Brain Behav Immun 22: 1248-1256.

Iijima I, Minamikawa J, Jacobson AE, Brossi A, Rice KC (1978). Studies in the $(+)$-morphinan series. 5. Synthesis and biological properties of (+)-naloxone. J Med Chem 21: 398-400.

Kalivas PW (1993). Neurotransmitter regulation of dopamine neurons in the ventral tegmental area. Brain Res Brain Res Rev 18: 75-113.

Koo JW, Mazei-Robison MS, Chaudhury D, Juarez B, LaPlant Q, Ferguson D et al (2012). BDNF is a negative modulator of morphine action. Science 338: 124-128.

Koob GF, Le Moal M (2008). Addiction and the brain antireward system. Annu Rev Psychol 59: 29-53.

Kosten TA, Marby DW, Nestler EJ (1991). Cocaine conditioned place preference is attenuated by chronic buprenorphine treatment. Life Sci 49: P1201-Pl206.

Kuzmin AV, Gerrits MA, van Ree JM, Zvartau EE (1997). Naloxone inhibits the reinforcing and motivational aspects of cocaine addiction in mice. Life Sci 60: Pl-257-Pl-264.

Laviolette SR, van der Kooy D (2004). GABAA receptors signal bidirectional reward transmission from the ventral tegmental area to the tegmental pedunculopontine nucleus as a function of opiate state. Eur J Neurosci 20: 2179-2187.

Leal G, Afonso PM, Salazar IL, Duarte CB (2014). Regulation of hippocampal synaptic plasticity by BDNF. Brain Res (e-pub ahead of print 23 October 2014).

Leri F, Flores J, Rajabi H, Stewart J (2003). Effects of cocaine in rats exposed to heroin. Neuropsychopharmacology 28: 2102-2116.

Leri F, Zhou Y, Goddard B, Cummins E, Kreek MJ (2006). Effects of high-dose methadone maintenance on cocaine place conditioning, cocaine self-administration, and mu-opioid receptor mRNA expression in the rat brain. Neuropsychopharmacology 31: 1462-1474.

Lewis SS, Loram LC, Hutchinson MR, Li CM, Zhang Y, Maier SF et al (2012). (+)-Naloxone, an opioid-inactive toll-like receptor 4 signaling inhibitor, reverses multiple models of chronic neuropathic pain in rats. J Pain 13: 498-506.

Lu L, Dempsey J, Liu SY, Bossert JM, Shaham Y (2004). A single infusion of brain-derived neurotrophic factor into the ventral 
tegmental area induces long-lasting potentiation of cocaine seeking after withdrawal. J Neurosci 24: 1604-1611.

Madhavan A, Bonci A, Whistler JL (2010). Opioid-Induced GABA potentiation after chronic morphine attenuates the rewarding effects of opioids in the ventral tegmental area. J Neurosci 30: 14029-14035.

Mattioli TA, Leduc-Pessah H, Skelhorne-Gross G, Nicol CJ, Milne B, Trang $\mathrm{T}$ et al (2014). Toll-like receptor 4 mutant and null mice retain morphine-induced tolerance, hyperalgesia, and physical dependence. PloS ONE 9: e97361.

Raghavendra V, Tanga FY, DeLeo JA (2004). Attenuation of morphine tolerance, withdrawal-induced hyperalgesia, and associated spinal inflammatory immune responses by propentofylline in rats. Neuropsychopharmacology 29: 327-334.

Rivera C, Li H, Thomas-Crusells J, Lahtinen H, Viitanen T, Nanobashvili A et al (2002). BDNF-induced TrkB activation down-regulates the $\mathrm{K}+-\mathrm{Cl}$ - cotransporter $\mathrm{KCC} 2$ and impairs neuronal Cl- extrusion. J Cell Biol 159: 747-752.

Rivera C, Voipio J, Payne JA, Ruusuvuori E, Lahtinen H, Lamsa K et al (1999). The $\mathrm{K}+/ \mathrm{Cl}$ - co-transporter $\mathrm{KCC} 2$ renders GABA hyperpolarizing during neuronal maturation. Nature 397: 251-255.

Seroogy KB, Lundgren KH, Tran TM, Guthrie KM, Isackson PJ, Gall CM (1994). Dopaminergic neurons in rat ventral midbrain express brain-derived neurotrophic factor and neurotrophin3 mRNAs. J Comp Neurol 342: 321-334.

Stevenson GW, Wentland MP, Bidlack JM, Mello NK, Negus SS (2004). Effects of the mixed-action kappa/mu opioid agonist 8-carboxamidocyclazocine on cocaine- and food-maintained responding in rhesus monkeys. Eur J Pharmacol 506: 133-141.

Taylor AM, Castonguay A, Taylor AJ, Murphy NP, Ghogha A, Cook C et al (2015). Microglia disrupt mesolimbic reward circuitry in chronic pain. J Neurosci 35: 22.

Taylor SR, Badurek S, Dileone RJ, Nashmi R, Minichiello L, Picciotto MR (2014). GABAergic and glutamatergic efferents of the mouse ventral tegmental area. J Comp Neurol 522: 3308-3334.

Ting AKR, Vargas-Perez H, Bufalino MR, Bahi A, Dreyer JL, Tyndale RF et al (2013). Infusion of brain-derived neurotrophic factor into the ventral tegmental area switches the substrates mediating ethanol motivation. Eur J Neurosci 37: 996-1003.

Vargas-Perez H, Bahi A, Bufalino MR, Ting AKR, Maal-Bared G, Lam J et al (2014). BDNF signaling in the VTA links the drug-dependent state to drug withdrawal aversions. J Neurosci 34: 7899-7909.

Vargas-Perez H, Ting AKR, Walton CH, Hansen DM, Razavi R, Clarke L et al (2009). Ventral tegmental area BDNF induces an opiate-dependent-like reward state in naïve rats. Science 324: 1732-1734.

Viitanen T, Ruusuvuori E, Kaila K, Voipio J (2010). The K+-Cl cotransporter KCC2 promotes GABAergic excitation in the mature rat hippocampus. J Physiol 588(Pt 9): 1527-1540.

Wang Q, Zhou H, Gao H, Chen SH, Chu CH, Wilson B et al (2012). Naloxone inhibits immune cell function by suppressing superoxide production through a direct interaction with gp9lphox subunit of NADPH oxidase. J Neuroinflamm 9: 32.

Weiss F, Koob GF (2001). Drug addiction: functional neurotoxicity of the brain reward systems. Neurotox Res 3: 145-156.

Williams JT, Ingram SL, Henderson G, Chavkin C, von Zastrow M, Schulz S et al (2013). Regulation of mu-opioid receptors: desensitization, phosphorylation, internalization, and tolerance. Pharmacol Rev 65: 223-254.

Zhang Y, Picetti R, Butelman ER, Schlussman SD, Ho A, Kreek MJ (2009). Behavioral and neurochemical changes induced by oxycodone differ between adolescent and adult mice. Neuropsychopharmacology 34: 912-922.

This work is licensed under a Creative Commons Attribution 4.0 International License. The images or other third party material in this article are included in the article's Creative Commons license, unless indicated otherwise in the credit line; if the material is not included under the Creative Commons license, users will need to obtain permission from the license holder to reproduce the material. To view a copy of this license, visit http://creativecommons.org/licenses/ by/4.0/

Supplementary Information accompanies the paper on the Neuropsychopharmacology website (http://www.nature.com/npp) 